\title{
BMJ Lifetime risk of developing coronary Open heart disease in Aboriginal Australians: a cohort study
}

\author{
Zhiqiang Wang, Wendy E Hoy
}

To cite: Wang Z, Hoy WE. Lifetime risk of developing coronary heart disease in Aboriginal Australians: a cohort study. BMJ Open 2013;3: 002308 .

doi:10.1136/bmjopen-2012002308

- Prepublication history and additional material for this paper are available online. To view these files please visit the journal online (http://dx.doi.org/10.1136/ bmjopen-2012-002308).

Received 6 November 2012 Revised 27 December 2012 Accepted 10 January 2013

This final article is available for use under the terms of the Creative Commons Attribution Non-Commercial 2.0 Licence; see http://bmjopen.bmj.com

Centre for Chronic Disease, School of Medicine, University of Queensland, Herston, Australia

Correspondence to Dr Zhiqiang Wang; z.wang@uq.edu.au

\section{ABSTRACT}

Objectives: Lifetime risk of coronary heart disease (CHD) is an important yardstick by which policy makers, clinicians and the general public can assess and promote the awareness and prevention of $\mathrm{CHD}$. The lifetime risk in Aboriginal people is not known. Using a cohort with up to 20 years of follow-up, we estimated the lifetime risk of CHD in Aboriginal people.

Design: A cohort study.

Setting: A remote Aboriginal region.

Participants: 1115 Aboriginal people from one remote tribal group who were free from $\mathrm{CHD}$ at baseline were followed for up to 20 years.

Main outcome measures: During the follow-up period, new CHD incident cases were identified through hospital and death records. We estimated the lifetime risks of $\mathrm{CHD}$ with and without adjusting for the presence of competing risk of death from non-CHD causes.

Results: Participants were followed up for 17126 person-years, during which 185 developed CHD and 144 died from non-CHD causes. The average age at which the first CHD event occurred was 48 years for men and 49 years for women. The risk of developing $\mathrm{CHD}$ increased with age until 60 years and then decreased with age. Lifetime cumulative risk without adjusting for competing risk was $70.7 \%$ for men and $63.8 \%$ for women. Adjusting for the presence of competing risk of death from non-CHD causes, the lifetime risk of $\mathrm{CHD}$ was $52.6 \%$ for men and $49.2 \%$ for women.

Conclusions: Lifetime risk of $\mathrm{CHD}$ is as high as one in two in both Aboriginal men and women. The average age of having first $\mathrm{CHD}$ events was under 50 years, much younger than that reported in nonAboriginal populations. Our data provide useful knowledge for health education, screening and prevention of $\mathrm{CHD}$ in Aboriginal people.

\section{INTRODUCTION}

The lifetime risk of a condition is defined as the probability that a person who is currently free of the condition will acquire it at some time during the remainder of their expected lifetime. ${ }^{1}$ It can be used to define risk from

\section{ARTICLE SUMMARY}

Article focus

- With long-term follow-up data, we estimated the lifetime CHD risk for Aboriginal men and women with adjustment for the presence of competing risk of death from non-CHD causes.

\section{Key messages}

- Lifetime risk is high in Aboriginal men and women, with one in two developing coronary heart disease (CHD) during their lifetime.

- The average age at which the first CHD event occurs is under 50 years (48 years for men and 49 for women), which is much younger than those reported in other populations (65 years in men and 70 years in women in the Framingham Study).

- Unlike in some other populations, the female population is not protective against $\mathrm{CHD}$ in this group of Aboriginal people. Aboriginal women have a similar lifetime CHD risk as their male counterparts.

Strengths and limitations of this study

- The major strengths of the study include the long-term follow-up and the high response rate.

- Major limitations include the following.

- Because this is a single region-based study, the generalisability of the findings needs to be further assessed.

- As CHD events were determined based on hospital records and under-reporting was possible, the lifetime risks in this study might have underestimated the true lifetime risk of the study population.

either birth or from a specific age. The lifetime risk estimates of coronary heart disease $(\mathrm{CHD})^{2}$ have been widely publicised to promote the public awareness, screening and prevention of CHD disease. We have shown that Aboriginal Australians in one remote region have a higher risk of CHD than that predicted using the Framingham function. ${ }^{3}$ They also have different levels of CHD risk factors from the general Australian population. ${ }^{4}$ The lifetime risks of CHD in Aboriginal 
men and women are still not known, even though CHD incidence rates have been reported in several studies. ${ }^{35-7}$ At an individual level, a person should be aware of the risk of CHD at any point in their life; similarly, at the population level, such lifetime risks are essential for public health planners to estimate the projected CHD burden with a long-term perspective in specific populations. Estimating lifetime risk requires a long-term follow-up and a consideration of the competing risk of death from non-CHD causes. ${ }^{2}$ There is a compelling need to generate estimates of lifetime risk of CHD in Aboriginal people, so that we may inform the public, clinicians and policy makers to take appropriate clinical and public health measures accordingly. Using cohort data with up to 20 years of follow-up, we estimated the lifetime risk of CHD in Aboriginal people of one tribal group living in a remote region of the Northern Territory of Australia, who have been experiencing a lifestyle transition from a hunter gathering way of life to a sedentary lifestyle. In this cohort study, we also estimated the lifetime risk of CHD for Aboriginal men and women originally free from CHD events at different ages separately.

\section{METHODS}

\section{Participants and CHD events}

Participants were recruited from a remote tribal group living in an isolated island setting in the Northern Territory of Australia from 1992 to 1995 . Only people who self-identified as belonging to the group, who had two parents of the same tribal group, were included. In all, 1115 participants, aged 10-75 years and free from clinically apparent CHD at baseline, representing over $80 \%$ of those age groups in the region, were included in this study. Measurements of baseline variables were described previously. ${ }^{4-12}$ All participants were followed up until 31 May 2012. During the follow-up period, new CHD events were identified through hospital records using codes of the International Classification of Diseases (ICD 9 codes 410-414 and ICD 10 codes I20I25), including myocardial infarction (410, I21), angina pectoris (411, I20) and other ischaemic heart disease (413, 414, I22, I23, I24 and I25). Deaths and their causes during the follow-up period were determined through a list of death records maintained at the community clinics. Figure 1 shows the details of follow-up of the study participants. Only the first-ever CHD incidents (fatal or non-fatal) were included in the analysis. For those participants who reached a CHD event or died from non-CHD causes during the follow-up, their follow-up time was from the age of their initial screening visit to the age of the first CHD event or death. Others who survived the follow-up period were censored at 31 May 2012. Since the individual hospital registration numbers which we used to track study participants were unique throughout the Northern Territory, we were able to capture their hospitalisation records even if our study participants had moved outside the local region. The chance of being hospitalised outside the Northern Territory was extremely low, if any, for people in this remote isolated region. Hospital or death records were identified for 1010 of 1115 (91\%) study participants and those without hospital and death records were regarded as free from CHD during the follow-up period.

\section{Statistical analysis}

The data were partitioned into age bands of $<20,20-29$, $30-39,40-49,50-59,60-69$ and $70+$ years throughout the follow-up. For those whose age fell into two or more age bands during the follow-up period, their total follow-up time was subdivided and allocated into corresponding age bands as described by Clayton and Hills. ${ }^{13}$ We calculated incidence, cumulative incidence and lifetime risk. Cumulative incidence of CHD was estimated using the Kaplan-Meir product-limit method. For
Figure 1 Coronary heart disease (CHD) events and deaths from non-CHD causes between baseline and the end of follow-up.

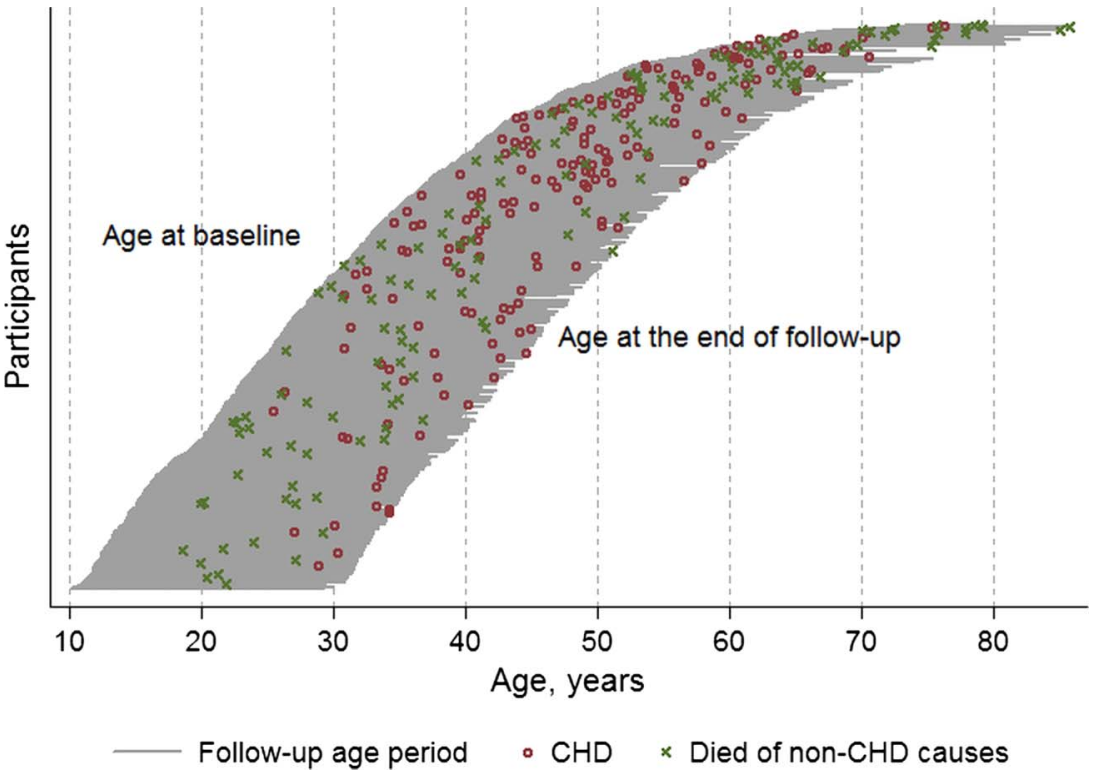


calculation of lifetime risk, we used a modified technique of survival analysis, and its computational technique has been described elsewhere in detail. ${ }^{14}{ }^{15}$ Briefly, the lifetime risk differs from the conventional cumulative risk estimated using the Kaplan-Meir method whenever there is a high risk of competing events. In this study, the competing events are the deaths from non-CHD causes which would remove people from being at risk of CHD. Although estimates of the theoretical cumulative risk assume that people who died of non-CHD causes would have developed CHD at the same rate as those who survived, estimates of the actual lifetime risk recognise that the risk of CHD after death is zero. ${ }^{5}$ We adjusted for the competing risk of death from non-CHD causes and calculated risk separately for men and women at each of the index ages of 30, 40, 50, 60 and 70 years. All analyses were done with Stata V.12.0. ${ }^{16}$

\section{RESULTS}

In all, 1115 participants who were free from CHD at baseline were followed up for 17126 person-years. During the follow-up period, 185 participants developed at least one CHD event, including 26 participants in whom the first CHD event was fatal. Among those who were free from CHD during the study period, 144 died from non-CHD causes. The mean age at the first CHD event was 48 for men and 49 for women. Tables 1 and 2 show the baseline characteristics of the study participants with different endpoints. Those who developed CHD events were older at baseline, and had higher levels of body mass index, blood pressure, cholesterol, triglycerides, urine albumin to creatin ratio and $\mathrm{C}$ reactive protein than those who did not develop CHD events. They also had higher prevalences at baseline of known diabetes, low estimated glomerular filtration rate, smoking and drinking than the non-CHD group.

The incidence rate of developing new onset CHD increased with age until 60 years and then decreased with age (table 3). In men, the incidence rate of CHD was less than $1 / 1000$ person-years for those under 30 years, increased to $5 / 1000$ person-years for those $30-39$ years and to $57 / 1000$ person-years for those $60-69$ years, but dropped to 21/1000 person-years after 70 years of age.

Theoretical cumulative risk without adjusting for competing risk of death from non-CHD causes was $71 \%$ (95\% CI 59 to 81 ) for men and 64\% (95\% CI 52 to 75 ) for women (figure 2). After adjusting for the presence of competing risk of death from non-CHD causes, the lifetime risk of CHD was 53\% (95\% CI 44 to 61) for men and $49 \%$ (95\% CI 40 to 57) for women.

We estimated the remaining lifetime risk of CHD adjusted for non-CHD deaths for those starting at 30, 40, 50,60 and 70 years (table 4). Since the numbers of participants aged 70 years or older were relatively small, the $95 \%$ CIs were wide. As expected, the remaining lifetime risk of a first CHD decreased as age free of CHD increased, from about one in two at 30 years to one in four in men or one in five in women at 70 years (figure 3 ).

\section{DISCUSSION}

Without adjusting for the competing effect of deaths from non-CHD causes, about two of three people in this cohort would theoretically suffer a CHD event in their lifetime. However, after adjusting for the presence of competing risk of deaths from non-CHD causes, the actual lifetime risk was estimated as one in two for both Aboriginal men and women.

The lifetime risk of CHD in this population was substantially lower than the theoretical cumulative risk. The

Table 1 Baseline characteristics of participants by CHD outcome: men

\begin{tabular}{|c|c|c|c|}
\hline & Non-CHD & CHD & Deaths from non-CHD causes \\
\hline Number & 408 & 98 & 85 \\
\hline Age (years) at baseline & $24.4(10.5)$ & $37.1(12.4)$ & $34.6(16.8)$ \\
\hline Age (years) at end* & $42.8(10.5)$ & $47.5(11.0)$ & $42.4(16.5)$ \\
\hline BMI $\left(\mathrm{kg} / \mathrm{m}^{2}\right)$ & $21.1(4.8)$ & $24.6(5.2)$ & $21.7(4.8)$ \\
\hline Waist circ $(\mathrm{cm})$ & $82.3(12.7)$ & $92.5(14.5)$ & $84.8(13.1)$ \\
\hline Systolic BP (mm Hg) & $120.4(15.0)$ & $128.0(21.5)$ & $128.2(18.1)$ \\
\hline Diastolic BP (mm Hg) & $71.9(11.9)$ & $82.2(16.8)$ & 79.2 (16.3) \\
\hline Total cholesterol (mmol/l) & $4.4(1.1)$ & $5.3(1.2)$ & $4.7(1.3)$ \\
\hline Triglycerides $(\mathrm{mmol} / \mathrm{l})$ & $1.9(1.4)$ & $2.8(1.9)$ & $2.6(3.3)$ \\
\hline $\mathrm{HDL}(\mathrm{mmol} / \mathrm{l})$ & $1.11(0.24)$ & $1.06(0.22)$ & $1.13(0.29)$ \\
\hline Urine ACR (mg/mmol) & $1.4(1.2,1.6)$ & $11.5(7.5,17.8)$ & $4.5(2.8,7.1)$ \\
\hline C reactive protein $(\mathrm{mg} / \mathrm{l})$ & $2.7(2.4,3.1)$ & $6.1(4.6,8.1)$ & $3.9(3.0,5.1)$ \\
\hline Smoking (n (\%)) & $232(56.9)$ & $69(70.4)$ & $57(67.1)$ \\
\hline Drinking (n (\%)) & $223(54.7)$ & $74(75.5)$ & $64(75.3)$ \\
\hline Known diabetes (n (\%)) & $11(2.7)$ & $22(22.4)$ & $8(9.4)$ \\
\hline Low eGFR (n (\%)) & 5 (1.3) & $9(9.6)$ & $7(8.2)$ \\
\hline
\end{tabular}

${ }^{*}$ Age at the time of the first CHD event, death from non-CHD causes or the end of study.

ACR, albumin to creatinine ratio, BMI, body mass index; BP, blood pressure; CHD, coronary heart disease, circ, circumference; eGFR, estimated Glomerular Filtration Rate, HDL, high-density lipoprotein. 
Table 2 Baseline characteristics of participants by CHD outcome: women

\begin{tabular}{|c|c|c|c|}
\hline & Non-CHD & CHD & Deaths from non-CHD causes \\
\hline Number & 378 & 87 & 59 \\
\hline Age (years) at baseline & $28.1(12.2)$ & $41.5(13.0)$ & $45.3(16.1)$ \\
\hline Age (years) at end* & $46.1(12.0)$ & $49.1(11.3)$ & $53.8(16.3)$ \\
\hline $\mathrm{BMI}\left(\mathrm{kg} / \mathrm{m}^{2}\right)$ & $23.3(6.0)$ & $26.1(6.0)$ & $22.3(6.1)$ \\
\hline Waist circ $(\mathrm{cm})$ & $88.6(14.6)$ & $96.8(13.0)$ & $89.8(15.0)$ \\
\hline Systolic BP (mm Hg) & $113.3(16.3)$ & $124.7(20.5)$ & $120.9(22.0)$ \\
\hline Diastolic BP (mm Hg) & $67.9(12.9)$ & $76.0(14.1)$ & $72.6(15.4)$ \\
\hline Total cholesterol (mmol/l) & $4.1(1.0)$ & $5.0(1.1)$ & $4.5(1.0)$ \\
\hline Triglycerides (mmol/l) & $1.9(1.3)$ & $2.7(1.6)$ & $2.0(1.1)$ \\
\hline $\mathrm{HDL}(\mathrm{mmol} / \mathrm{l})$ & $1.06(0.25)$ & $0.98(0.18)$ & $1.09(0.26)$ \\
\hline Urine ACR (mg/mmol) & $2.7(2.2,3.2)$ & $19.4(12.3,30.4)$ & $17.4(10.4,29.3)$ \\
\hline $\mathrm{C}$ reactive protein (mg/l) & $4.7(4.1,5.4)$ & $8.3(6.6,10.4)$ & $8.6(6.3,11.8)$ \\
\hline Smoking (n (\%) & $196(51.9)$ & $59(67.8)$ & $48(81.4)$ \\
\hline Drinking n (\%)) & $93(24.6)$ & $25(28.7)$ & $22(37.3)$ \\
\hline Known diabetes (n (\%)) & $19(5.0)$ & $25(28.7)$ & $10(16.9)$ \\
\hline Low eGFR (n (\%)) & $4(1.2)$ & $11(14.7)$ & $12(23.5)$ \\
\hline
\end{tabular}

${ }^{*}$ Age at the time of the first $\mathrm{CHD}$ event, death from non-CHD causes or the end of study.

ACR, albumin to creatinine ratio, BMI, body mass index; BP, blood pressure; CHD, coronary heart disease, eGFR, estimated Glomerular Filtration Rate, HDL, high-density lipoprotein.

conventional calculation of theoretical cumulative risk in this study was based on the assumption that people who died of non-CHD causes would have had the same rate as those who had survived, while estimates of lifetime risk or remaining lifetime risk recognised the fact that their risk of CHD after death was zero. The high competing risk of death from non-CHD causes in the study population and shorter life expectancies explain the substantially lower value in lifetime risk than that of the theoretical cumulative risk. Even so, the lifetime risks of CHD in Aboriginal people, particularly in Aboriginal women, were still higher than those reported in some other populations. ${ }^{45}$ The lifetime risk of CHD estimated in the Framingham Study was one in two in men and one in three in women. Only a small proportion of participants reached 75 years in our study, whereas a large proportion of study participants in the Framingham Study reached much older ages.

The average age among those having the first CHD events was much lower in these Aboriginal people than that reported in the non-Aboriginal populations. It was around 50 years in Aboriginal people, which is over 15 years earlier than in American men (65 years) and 20 years earlier than in American women (70 years). ${ }^{17}$ Unlike in some of the other populations in which the female gender is protective against CHD or cardiovascular disease, ${ }^{2}{ }^{18}$ Aboriginal women in this cohort had a similar lifetime risk as their male counterparts. Such a

Table 3 Incidence rates by age and sex in Aboriginal people

\begin{tabular}{|c|c|c|c|c|c|}
\hline Age (years) & Person-years & Number of participants & Number of CHD & Rate (/1000 person-years) & $95 \% \mathrm{Cl}$ \\
\hline \multicolumn{6}{|l|}{ Male } \\
\hline $10-$ & 985.5 & 184 & 0 & 0 & \\
\hline $20-$ & 2617.1 & 367 & 2 & 0.8 & 0.2 to 3.1 \\
\hline $30-$ & 2782.0 & 444 & 22 & 7.9 & 5.2 to 12.0 \\
\hline $40-$ & 1733.9 & 295 & 36 & 20.8 & 15.0 to 28.8 \\
\hline $50-$ & 722.5 & 143 & 22 & 30.4 & 20.0 to 46.2 \\
\hline $60-$ & 245.2 & 55 & 14 & 57.1 & 33.8 to 96.4 \\
\hline $70+$ & 94.9 & 18 & 2 & 21.1 & 5.3 to 84.3 \\
\hline \multicolumn{6}{|l|}{ Female } \\
\hline $10-$ & 594.0 & 115 & 0 & 0 & \\
\hline $20-$ & 1855.1 & 255 & 2 & 1.1 & $0.3,4.3$ \\
\hline $30-$ & 2207.3 & 346 & 18 & 8.2 & $5.1,12.9$ \\
\hline $40-$ & 1716.6 & 286 & 28 & 16.3 & $11.3,23.6$ \\
\hline $50-$ & 1019.2 & 181 & 24 & 23.5 & $15.8,35.1$ \\
\hline $60-$ & 383.2 & 87 & 11 & 28.7 & $15.9,51.8$ \\
\hline $70+$ & 169.3 & 29 & 4 & 23.6 & $8.9,63.0$ \\
\hline
\end{tabular}

$\mathrm{CHD}$, coronary heart disease; pys, person-years. 
Figure 2 Lifetime risk of coronary heart disease in Aboriginal people.

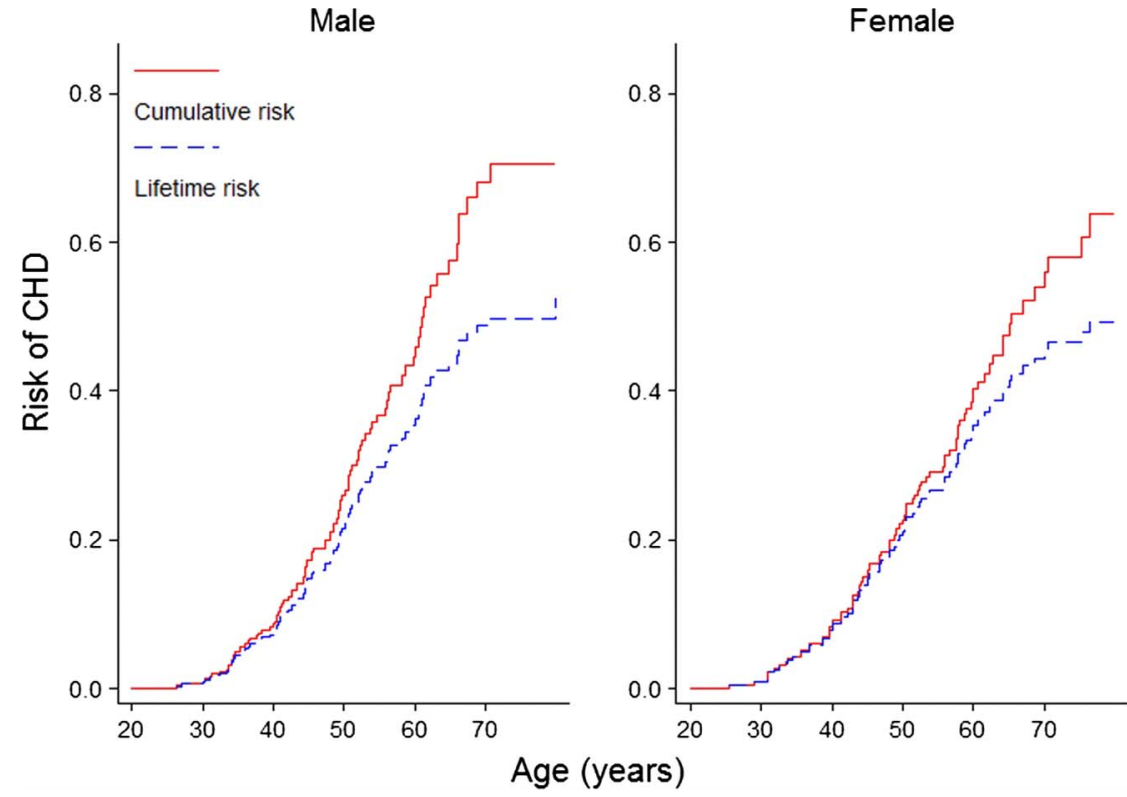

phenomenon of females having equal or higher risk has also been observed in Asian Indians. ${ }^{19}$

Lifetime risk and remaining lifetime risk estimates at different ages are more easily understood than incidence rates by the general public. Lifetime risks of CHD estimated in the Framingham Study have often been publicised as headline figures in the media and have increased public awareness of, and interest in, the importance and prevention of CHD. ${ }^{2}{ }^{20}$ In this study, the lifetime risks of CHD have been estimated for the first time in Aboriginal people. Those estimates can be useful for promoting public interest in prevention of CHD, particularly in Aboriginal younger people and women. Our lifetime and remaining lifetime risk estimates can also be used to guide the allocation of resources to improve public health services for CHD in this population. They may improve the preventive efforts from both clinicians and the general public. For example, in other settings, it was found that providing lifetime risk improved the prescription of aspirin or lipid-lowering medication. ${ }^{21}$ It has been anticipated that the lifetime risk will be incorporated in the next generation of guidelines related to medical therapy and lifestyle interventions for cardiovascular disease, as it should

Table 4 Lifetime risk of a first CHD event at different ages reached free of $\mathrm{CHD}$ in Aboriginal men and women

\begin{tabular}{lll}
\hline \multirow{2}{*}{ Age (years) } & \multicolumn{2}{l}{ Lifetime risk (95\% Cl) } \\
\cline { 2 - 3 } & Men & Women \\
\hline 30 & $58 \%(48$ to 67$)$ & $50 \%(42$ to 59$)$ \\
40 & $58 \%(47$ to 68$)$ & $48 \%(39$ to 57$)$ \\
50 & $54 \%(40$ to 66$)$ & $40 \%(30$ to 51$)$ \\
60 & $45 \%(27$ to 62$)$ & $31 \%(18$ to 44$)$ \\
$70+$ & $27 \%(4$ to 60$)$ & $20 \%(6$ to 39$)$ \\
\hline CHD, coronary heart disease.
\end{tabular}

impact positively on conveyance of CHD risk to the public. ${ }^{17}$ In this study, we started to provide some epidemiological evidence for developing relevant guidelines for Aboriginal people.

The use of lifetime risks in the clinical setting has been debated recently, ${ }^{22-24}$ particularly for individual risk prediction. First, lifetime risks do not distinguish between immediate and remote risks, and should be used in combination with short-term risk estimates such as incidence rates and 10-year risks when counselling young people. ${ }^{1}$ Second, lifetime risk levels are heavily driven by the remaining life expectancy owing to the competing risk of deaths from non-CHD causes. Shorter life expectancy will result in a lower lifetime risk, even though the incidence rate of CHD is high during the lifetime and at a specific time point. For example, the lifetime risk of one in two for Aboriginal men in our study is similar to the lifetime risk for non-Aboriginal men reported in the Framingham Study, ${ }^{2}$ but our estimates were mainly based on data from those participants who were under 75 years, while the estimates in the Framingham Study were based on data from those under 95 years. Therefore, when we compare the remaining lifetime CHD risks between two populations, their differences in life expectancy must be taken into consideration. The lifetime risk up to 50 years of age for Aboriginal people in this study is equivalent to that of non-Aboriginal people up to 70 years in men and up to 80 years in women in the Framingham Study. ${ }^{2}$

There are several limitations in the present study. First, we obtained the follow-up data from one tribal group in a remote Aboriginal region in the Northern Territory of Australia. It is possible that CHD risks are heterogeneous among different regions. It remains to be verified if the findings are generalisable to the broader Aboriginal population in Australia. Second, our lifetime risk estimates represent average values for a specific population. 
Figure 3 Lifetime risk of coronary heart disease for Aboriginal men and women for baseline ages of $30,40,50,60$ and 70 years reached free from coronary heart disease.

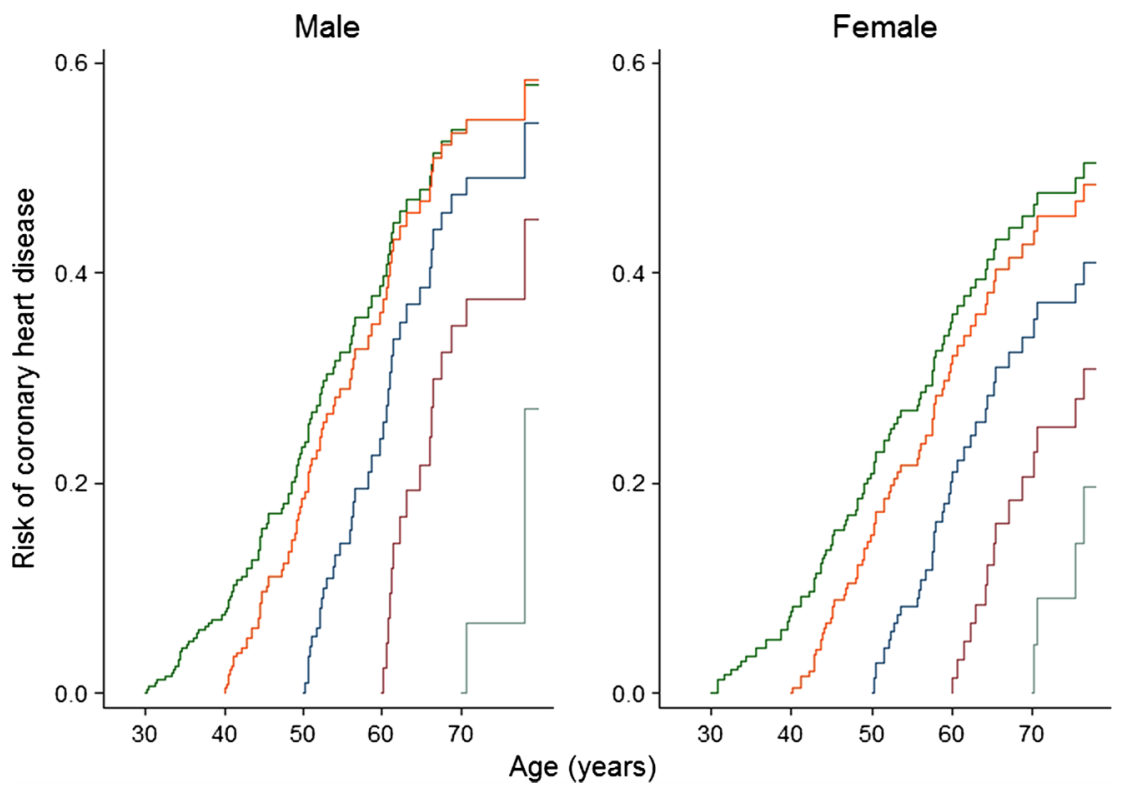

Lifetime risks vary according to the risk factor levels. $^{18}{ }^{25-28}$ In Aboriginal people, factors such as the urinary albumin-to-creatinine ratio, $\mathrm{C}$ reactive protein and obesity have been reported to be associated with CHD and mortality. 891229 Owing to a relatively small sample size, we did not calculate risk factor-specific lifetime risks in this study. Further investigation is needed as we collect more data with longer follow-up. Third, as the participants entered at different age points and they were not followed from birth (figure 1), cohort effects might have existed. The 70-year-old man today could have a different CHD risk from that of a 20 -year-old man when he reaches the age of 70 years, 50 years later. Finally, CHD events were determined based on routinely documented diagnosis information in hospital records during the follow-up period. Under-reporting is possible as some participants with minor CHD events might not have been hospitalised or not diagnosed as such. Therefore, lifetime risks in this study might have underestimated the true lifetime risk in the study population.

In summary, even adjusting for the high competing risk of deaths from non-CHD causes, Aboriginal people still have a high lifetime risk of CHD, and one-in-two men and women will have $\mathrm{CHD}$ during their lifetime. The average age of having first CHD events was under 50 years, much younger than that reported in non-Aboriginal populations. The female gender protective against CHD observed in other populations does not exist in Aboriginal people as the risk of CHD in Aboriginal women is just as high as that in their men counterparts.

Acknowledgements We especially thank the Aboriginal people who participated in this study. The baseline data were collected by the renal research team led by WEH at the Menzies School of Health Research, Darwin, Australia. Shuqin Li at the Northern Territory Department of Health assisted in the interpretation of hospital data.

Contributors WEH and ZW conceived the idea of the study and were responsible for the design of the study. WEH provided input to the data analysis and was responsible for the acquisition of the baseline data. ZW was responsible for linking baseline and hospital data and for undertaking the data analysis. Both WEH and ZW contributed to the first draft, and read and approved the final version.

Funding This work was supported by NHMRC of Australia (APP1025300). WEH was supported by an Australian Fellowship (\#511081) and ZW by an NHMRC Fellowship (\#511013 \& APP1042343).

Competing interests None.

Ethics approval The project was approved by the University of Queensland Behavioural \& Social Science Ethical Review Committee (\#2011001232).

Provenance and peer review Not commissioned; externally peer reviewed. Data sharing statement There are no additional data available.

\section{REFERENCES}

1. Seshadri S, Wolf PA. Lifetime risk of stroke and dementia: current concepts, and estimates from the Framingham Study. Lancet Neurol 2007;6:1106-14.

2. Lloyd-Jones DM, Larson MG, Beiser A, et al. Lifetime risk of developing coronary heart disease. Lancet 1999;353:89-92.

3. Wang Z, Hoy WE. Is the Framingham coronary heart disease absolute risk function applicable to Aboriginal people? Med J Aust 2005;182:66-9.

4. Wang Z, Hoy WE. Hypertension, dyslipidemia, body mass index, diabetes and smoking status in Aboriginal Australians in a remote community. Ethn Dis 2003;13:324-30. events in Aboriginal Australians: incidence in an urban population. Med J Aust 2009;190:583-6.

6. McDermott RA, McCulloch B, Li M. Glycaemia and albuminuria as predictors of coronary heart disease in Aboriginal and Torres Strait Islander adults: a north Queensland cohort. Med J Aust 2011;194:514-18.

7. Rowley KG, O'Dea K, Anderson I, et al. Lower than expected morbidity and mortality for an Australian Aboriginal population: 10 -year follow-up in a decentralised community. Med $\mathrm{J}$ Aust 2008;188:283-7.

8. McDonald SP, Wang Z, Hoy WE. Physical and biochemical predictors of death in an Australian Aboriginal cohort. Clin Exp Pharmacol Physiol 1999;26:618-21.

9. Hoy WE, Wang Z, VanBuynder $P$, et al. The natural history of renal disease in Australian aborigines. Part 1. Changes in albuminuria and glomerular filtration rate over time. Kidney Int 2001;60:243-8.

10. Wang Z, Hoy WE. Waist circumference, body mass index, hip circumference and waist-to-hip ratio as predictors of cardiovascular disease in Aboriginal people. Eur J Clin Nutr 2004;58:888-93.
5. Bradshaw PJ, Alfonso HS, Finn JC, et al. Coronary heart disease 
11. Wang Z, Hoy WE. Albuminuria and incident coronary heart disease in Australian Aboriginal people. Kidney Int 2005;68:1289-93.

12. Wang Z, Hoy WE. C-reactive protein: an independent predictor of cardiovascular disease in Aboriginal Australians. Aust N Z J Public Health 2010;34(Suppl 1):S25-9.

13. Clayton D, Hills M. Statistical models in epidemiology. Oxford: Oxford University Press, 1993.

14. Seshadri S, Wolf PA, Beiser A, et al. Lifetime risk of dementia and Alzheimer's disease. The impact of mortality on risk estimates in the Framingham Study. Neurology 1997;49:1498-504.

15. Beiser A, D'Agostino RB Sr., Seshadri S, et al. Computing estimates of incidence, including lifetime risk: Alzheimer's disease in the Framingham Study. The practical incidence estimators (PIE) macro. Stat Med 2000;19:1495-522.

16. Stata Statistical Software: Release 12 (program). College Station, TX: StataCorp LP, 2011.

17. Bleakley C, Millar A, Harbinson M, et al. Lifetime risk of cardiovascular disease: the next generation in risk prediction. Can J Cardiol 2013;29:147-50.

18. Berry JD, Dyer A, Cai X, et al. Lifetime risks of cardiovascular disease. N Engl J Med 2012;366:321-9.

19. Jeemon P, Prabhakaran D, Huffman MD, et al. Distribution of 10-year and lifetime predicted risk for cardiovascular disease in the Indian Sentinel Surveillance Study population (cross-sectional survey results). BMJ Open 2011;1:e000068.

20. Roger VL, Go AS, Lloyd-Jones DM, et al. Heart disease and stroke statistics-2012 update: a report from the American Heart Association. Circulation 2012;125:e2-220.
21. Persell SD, Zei C, Cameron KA, et al. Potential use of 10-year and lifetime coronary risk information for preventive cardiology prescribing decisions: a primary care physician survey. Arch Intern Med 2010;170:470-7.

22. Jackson $\mathrm{R}$, Kerr A, Wells $\mathrm{S}$. Is estimating lifetime cardiovascular risk useful? BMJ 2010;341:c7379.

23. Sasieni PD. Utility of lifetime risks. In defence of lifetime risk. BMJ 2011;342:d1490.

24. Hippisley-Cox J, Coupland C, Robson J, et al. Derivation, validation, and evaluation of a new QRISK model to estimate lifetime risk of cardiovascular disease: cohort study using QResearch database. BMJ 2010;341:c6624.

25. Lloyd-Jones DM, Wilson PW, Larson MG, et al. Lifetime risk of coronary heart disease by cholesterol levels at selected ages. Arch Intern Med 2003;163:1966-72.

26. Allen N, Berry JD, Ning H, et al. Impact of blood pressure and blood pressure change during middle age on the remaining lifetime risk for cardiovascular disease: the cardiovascular lifetime risk pooling project. Circulation 2012;125:37-44.

27. Lloyd-Jones DM, Larson MG, Leip EP, et al. Lifetime risk for developing congestive heart failure: the Framingham Heart Study. Circulation 2002;106:3068-72.

28. Berry JD, Willis B, Gupta S, et al. Lifetime risks for cardiovascular disease mortality by cardiorespiratory fitness levels measured at ages 45,55 , and 65 years in men. The Cooper Center Longitudina Study. J Am Coll Cardiol 2011;57:1604-10.

29. Hoy WE, Wang $Z$, VanBuynder $P$, et al. The natural history of renal disease in Australian Aborigines. Part 2. Albuminuria predicts natura death and renal failure. Kidney Int 2001;60:249-56. 